\title{
CONGENITAL GYSTIC DISEASE OF THE LIVER IN SEVEN DOGS
}

\author{
By \\ T. S. G. A. M. VAN Den INGh and J. Rothuizen \\ Department of Veterinary Pathology and Department of Small Animal Medicine, State University Utrecht, \\ The Netherlands
}

\section{INTRODUGTION}

Congenital cysts of the liver, either solitary or multiple, have been reported in the dog (Jubb and Kennedy, 1970; McKenna and Carpenter, 1980; Jones and Hunt, 1983). In man, various forms of cystic disease of the liver are known (Hadad, Westbrook, Campbell, Caldwell and Morris, 1976; Ishak and Sharp, 1979).

The purpose of this article is to describe several cases of cystic liver disease in the dog and to discuss these cases and the canine cases in the literature in relation to the various forms of congenital cystic disease of the liver in man.

MATERIALS AND METHODS

\section{Case Reports}

In most cases the cystic liver lesions were coincidental findings. A direct relation between the cystic lesions and clinical disease existed in dogs 4 and 7 , and clinical signs, physical examination results, laboratory values and radiography will only be given for these dogs.

(1) Collie, 5 years old, female. At necropsy the dog showed lymphoid leucosis, septic thrombo-endocarditis of the bicuspid valves, and a large lipoma in the abdomen. A large unilocular cyst $10 \mathrm{~cm}$ in diameter was found in the right lateral liver lobe (Fig. 1). Histologically, the cyst was lined by a single layer of cuboidal or flattened epithelium and surrounded by mature connective tissue.

(2) Poodle, 11 years old, female. At necropsy the sole finding was a solitary liver cyst, measuring $4 \times 3 \times 3 \mathrm{~cm}$ at the caudal margin of the right lateral liver lobe. Histologically, a multilocular cyst was seen lined by cuboidal, columnar or flattened epithelium and surrounded by connective tissue, which locally was increased and showed a proliferation of small, often cystic, bile ducts.

(3) German Shepherd, 9 years old, castrated male. At necropsy, the animal showed a leiomyosarcoma of the small intestine with several large metastases in the liver. Coincidentally, a unilocular liver cyst measuring about $4 \mathrm{~cm}$ in diameter and filled with mucinous material was found in right medial liver lobe. Microscopically, the cyst had a single layer of cuboidal or columnar epithelium and was surrounded by dense fibrous tissue with minimal evidence of inflammation.

(4) Pekingese, 9 years old, female. The dog had been vomiting for one week. Physical examination revealed right-sided hepatomegaly. Blood examination showed no abnormalities. Values for urea, alkaline phosphatase (AP) and alanine transferase (ALT) were within normal limits. Radiographically, the left kidney appeared to be enlarged.

At necropsy a large unilocular cyst measuring $7 \times 6 \times 3 \mathrm{~cm}$ and some smaller ones were present in the right lateral liver lobe. In addition, several cysts measuring up to 
$1.5 \mathrm{~cm}$ in diameter were present throughout the cortex and medulla of both kidneys (Fig. 2). Histologically, the lining of the liver cysts consisted of cuboidal or columnar epithelium, surrounded by a layer of fibrous tissue merging into the adjacent portal areas. The large cyst also showed areas with flattened epithelium and with ulceration and inflammation. In the kidneys an intimate intermixture of normal and abnormal structures was present, with cystic dilatation of various parts of the nephrons including Bowmans' space.

(5) Dalmatian, 12 years old, male. At necropsy, the animal showed endocardiosis of the mitral valves with jet-lesions and dilatation of the left atrium suggesting mitral valve insufficiency, chronic interstitial nephritis with large concrements in the pelvis of both kidneys, a solitary cyst, $1 \mathrm{~cm}$ in diameter, in the left kidney and metastatic calcification and oedema of the lungs. Coincidentally, multiple cysts of about $1 \mathrm{~cm}$ in diameter were found throughout the liver. Histologically, these liver cysts were multilocular and lined by cuboidal or columnar epithelium (Fig. 3).

(6) English Cocker Spaniel, 5 years old, spayed. Necropsy revealed acromegaly, diabetes mellitus and chronic pyelonephritis.

As a coincidental finding, in the left lateral liver lobe some cysts measuring up to $1 \mathrm{~cm}$ were found; nearby was a small fibrous scar, $0.5 \mathrm{~cm}$ in diameter. Microscopically, the cysts were multilocular, lined by cuboidal or columnar epithelium and surrounded by a rim of fibrous tissue merging in to the adjacent portal areas. The scar consisted of a conglomerate of ducts embedded in a collagenous stroma directly related to the portal areas. The ducts were rounded or irregular, mostly dilated and lined by cuboidal or columnar epithelium (Fig. 4). These changes are consistent with a Von Meyenburg complex.

(7) Collie, 14 months old, male. The animal developed seizures and apathy over a period of one month and polydipsia for 2 months. At physical examination an abdominal mass near the left kidney was found. Blood examination revealed a marked uraemia and increased activity of AP and ALT. Radiographic examination revealed calcified structures, possibly within the biliary tract (Fig. 5). Exploratory laparotomy revealed a large, cyst-like structure near the gall-bladder from which $900 \mathrm{ml}$ of bile was aspirated and which was at once refilled with a radioopaque contrast fluid. Subsequent radiographs revealed the cyst to be part of a severely dilated extrahepatic bile duct system (Fig. 6). Because of the many abnormalities found during laparotomy the dog was destroyed.

At necropsy, the extrahepatic bile ducts were in position and patent. They showed severe and diffuse dilatation (Fig. 7) and contained a pale-yellow viscid fluid. The liver size was normal; the consistency was increased because of bridging fibrosis which gave the liver the appearance of a pig's liver. The cut surface of the liver showed small cysts regularly distributed. At micro-dissection these cysts were seen as diffusely dilated intrahepatic bile ducts; many bile concrements up to $4 \mathrm{~mm}$ in diameter were present in the dilated bile ducts and obstructed their lumen. The kidneys were normal in size and pale in colour. On the cut surface, fusiform radially arranged cysts and fibrosis were present throughout the cortex (Fig. 8).

Histologically, the liver was normal in structure except that the portal areas were enlarged and included increased mature connective tissue with multiple cross-sections of dilated bile ducts; sporadically, lymphoid cells were present (Fig. 9). The bile ducts had cylindrical or cuboidal epithelium without papillary infoldings; small and larger concrements were frequently seen in the lumen (Fig. 10), sometimes causing destruction of the epithelium and resultant focal inflammation. The limiting plate and the lobular parenchyma had a normal appearance. The renal cortex showed many cysts lined with flattened or cuboidal epithelium and massive interstitial fibrosis with islands of convoluted tubules and often solitary glomeruli. In the subcapsular region mononuclear infiltrates were seen. Except for moderate fibrosis, the medulla was normal. 

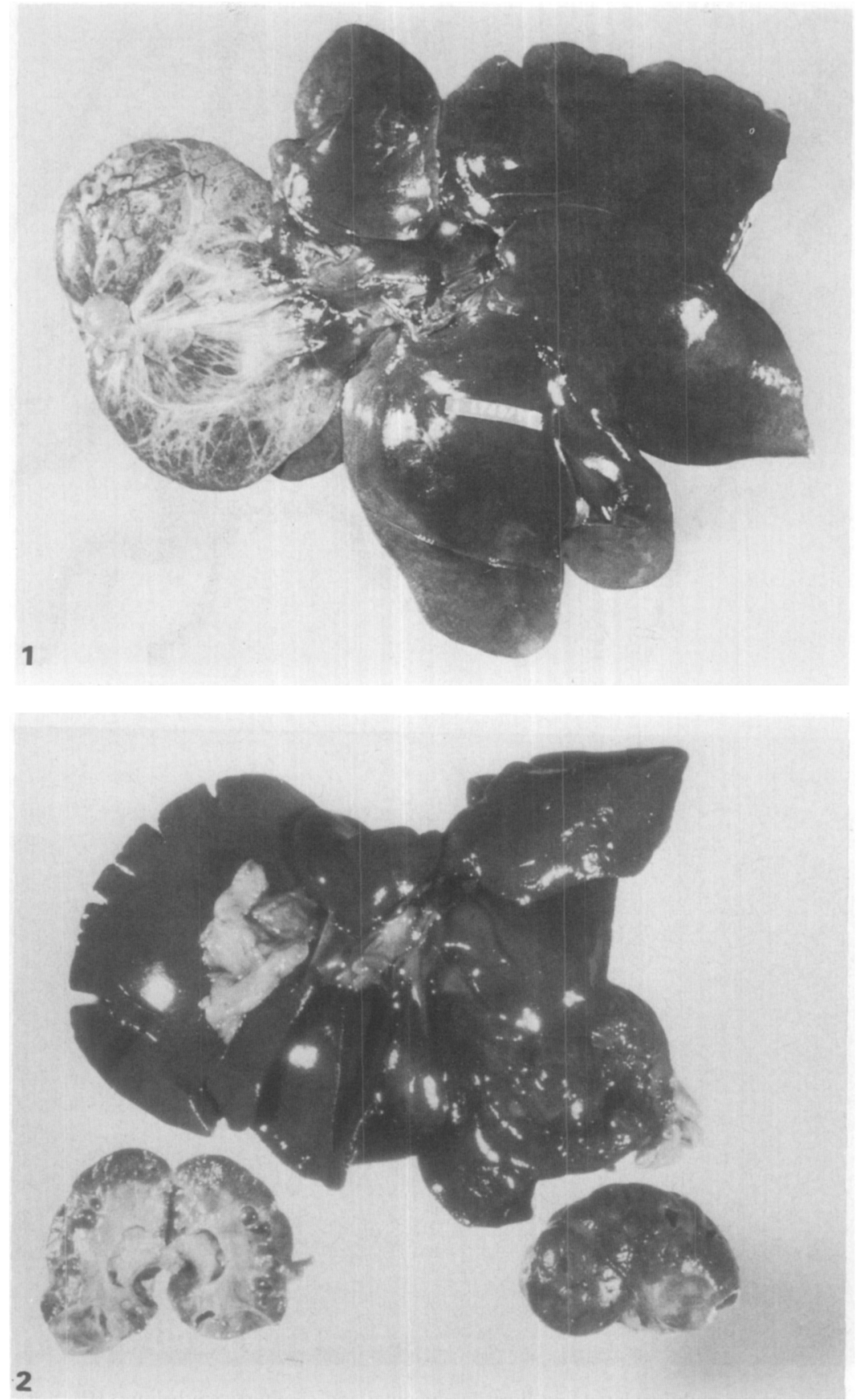

Fig. 1. Case 1. A large solitary cyst in the right lateral liver lobe.

Fig. 2. Case 4. Multiple cysts in the right lateral liver lobe and in the kidneys. 

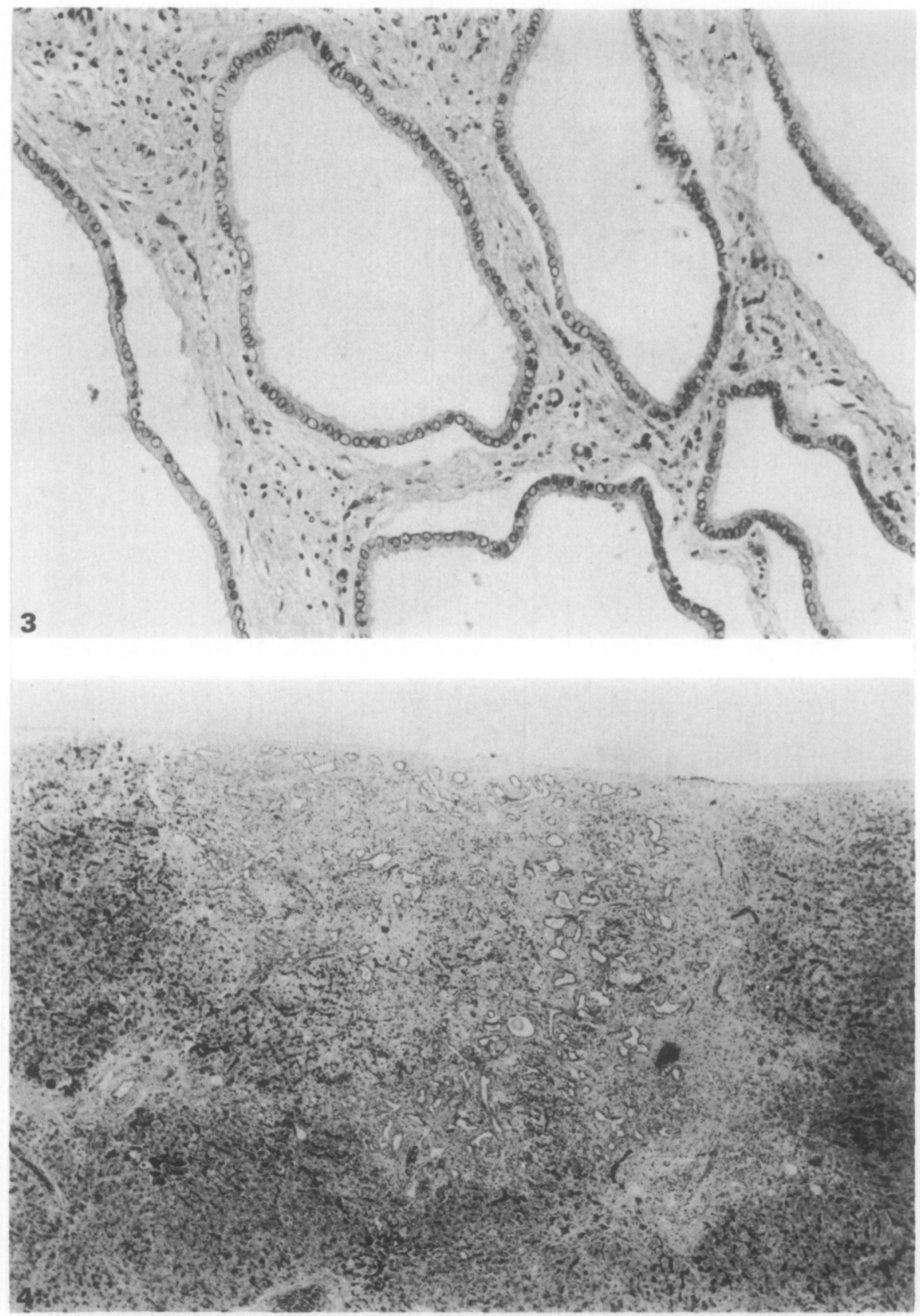

Fig. 3. Case 5. Liver: multiloculated cyst lined by cuboidal and columnar epithelium. $\mathrm{HE} \times 20$.

Fig. 4. Case 6. Liver: a Von Meyenburg complex. HE × 2.5. 

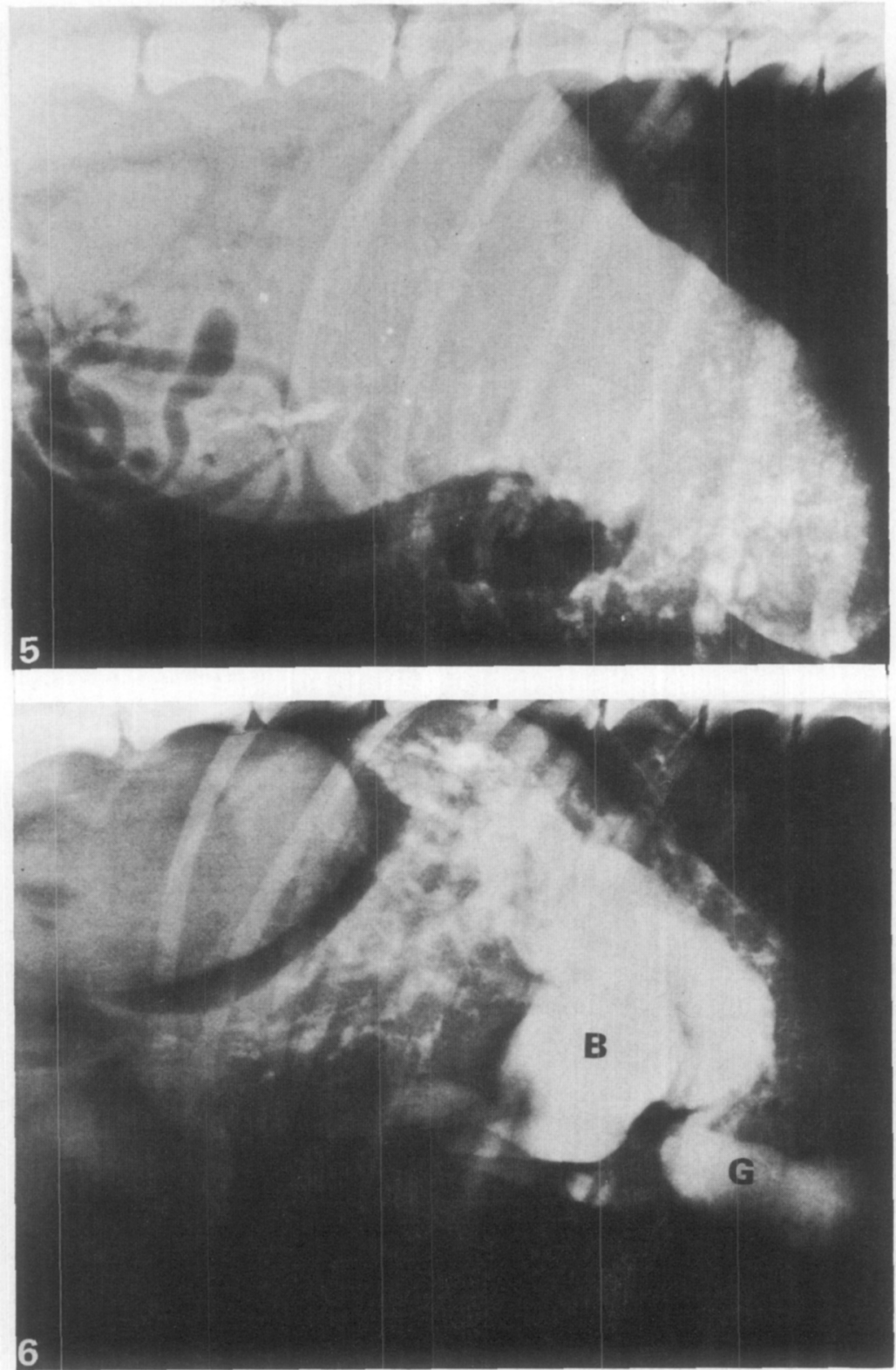

Fig. 5. Case 7. Plain lateral radiograph. Multiple calcifications in the region of the liver.

Fig. 6. Case 7. Lateral radiograph after intra-operative injection of radioopaque contrast fluid in the biliary system. Severely dilated extrahepatic bile ducts $(B), G=$ gall-bladder. 

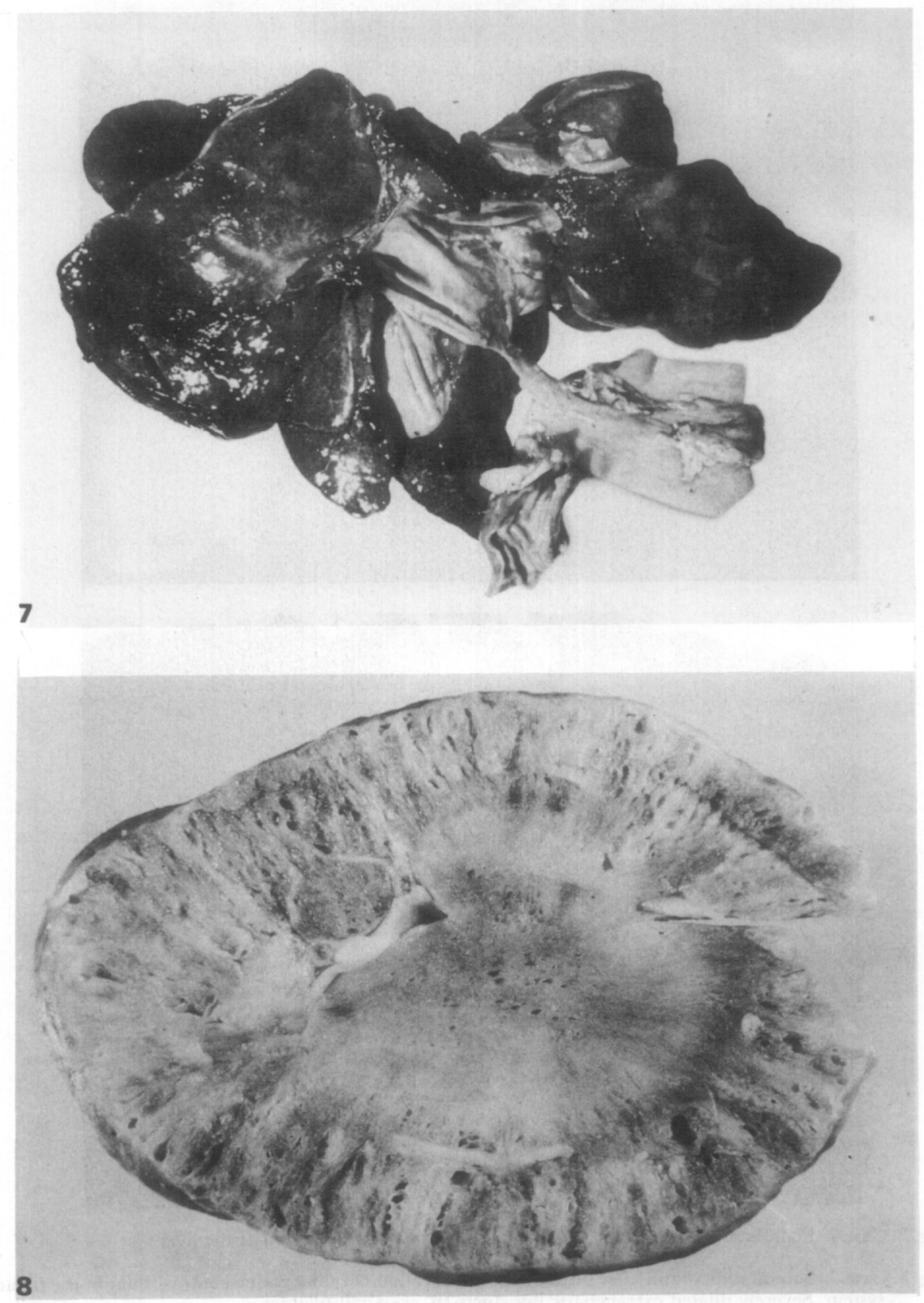

Fig. 7. Case 7. Liver: massive diffuse dilatation of the extrahepatic bile ducts.

Fig. 8. Case 7. Gut surface of the kidney. Fusiform radially arranged cysts and fibrosis, especially in the cortex. 

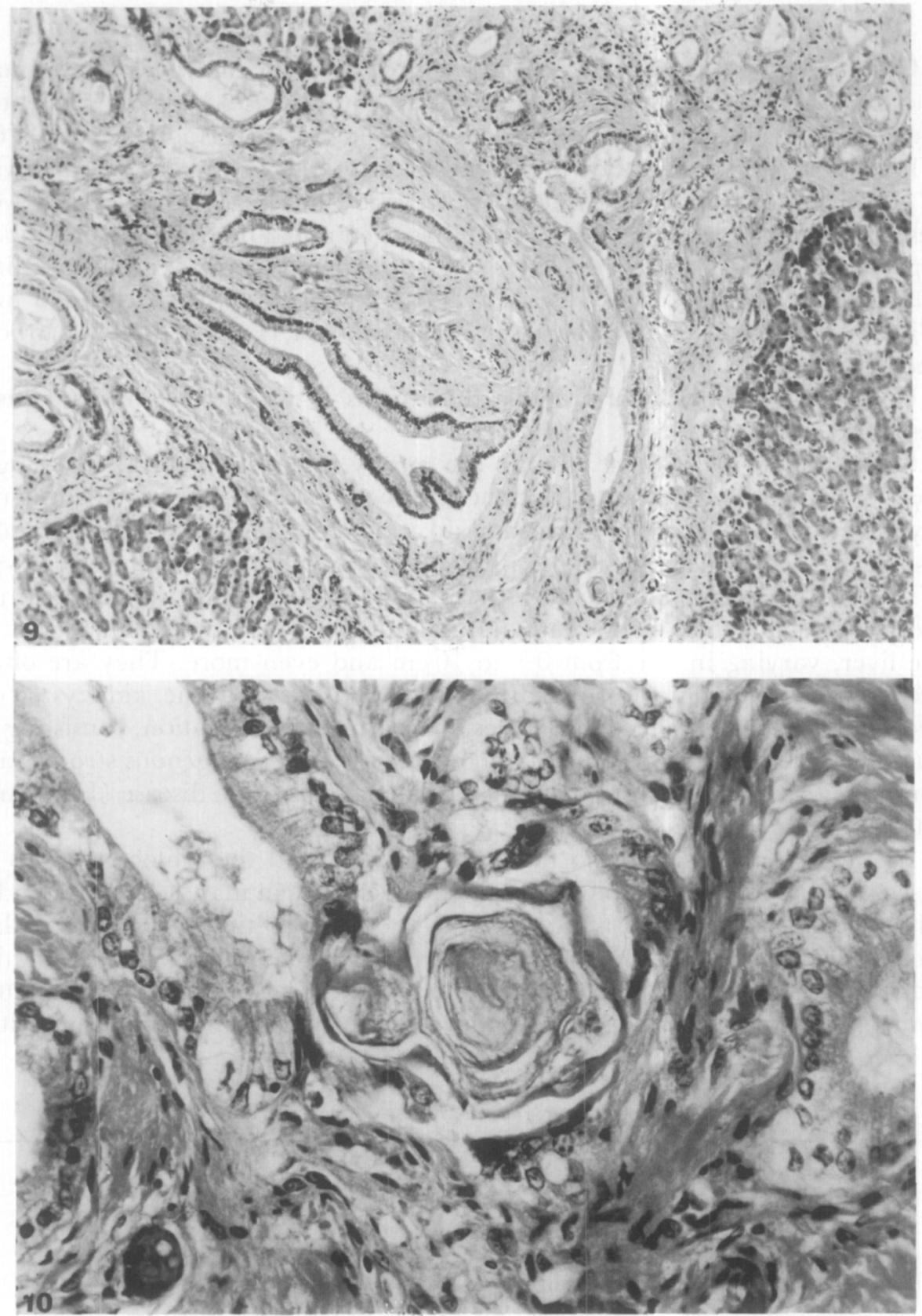

Fig. 9. Case 7. Liver: increase of portal connective tissue and multiple cross-sections of dilated bile ducts. HE $\times 20$.

Fig. 10. Case 7. Iiver: concrement in the lumen of a dilated bile duct. HE $\times 40$. 


\section{DISGUSSION}

Cystic disease of the liver has been a source of much controversy in human literature concerning the definition of the various diseases. A widely accepted classification is given in Table 1 (Ishak and Sharp, 1979; Hadad et al., 1976). Whereas congenital dilatation of the bile ducts types I, II and III, solitary cysts of the liver and the adult type of polycystic disease of the liver are well described entities, considerable overlap has been noted between the childhood type of polycystic disease of the liver, congenital hepatic fibrosis and congenital dilatation of the bile ducts types IV and V (Murray-Lyon, Ockenden and Williams, 1973; Ishak and Sharp, 1979; Nakanuma, Terada, Ohta, Kurachi and Matsubara, 1982).

Congenital dilatation of the bile duct types I, II and III has not been described in the dog.

Solitary cysts of the liver in man are mostly unilocular and have a single layer of cuboidal, columnar or flattcncd cpithclium. The cysts vary considcrably in size; they contain proteinacous fluid and mucin; blood and bilirubin may also be present. The aetiology and pathogenesis are not known. The adult type of polycystic disease of the liver in man is an autosomal dominant trait morphologically characterized by multiple unilocular or multilocular cysts in the liver, varying in size from 0.1 to $10 \mathrm{~cm}$ and even more. They are often accompanied by solitary or multiple, rounded cysts in the kidneys. Von Meyenburg complexes, i.e. discrete lesions, periportal in location, consisting of ducts with slightly dilated lumina and embedded in a collagenous stroma, are considered as part of the spectrum of adult type polycystic disease (Ishak and Sharp, 1979).

In our material the multilocular cyst in case 2 and the unilocular cysts in cases 1 and 3 are comparable with solitary liver cysts in man. Cases 4, 5 and 6 all showed multiple uni- or multilocular liver cysts, sometimes associated with a Von Meyenburg complex (case 6) or with multiple, rounded cysts in the kidneys (case 4). The solitary cyst in the kidney of case 5 may be associated with the liver cysts, or may be secondary to the chronic interstitial nephritis.

TABLE 1

CLASSIFICATION OF CONGENITAL GYSTIG DISEASE OF THE LIVER IN MAN

1. Solitary cyst of the liver

2. Polycystic disease of the liver

- childhood type

- adult type

3. Congenital hepatic fibrosis

4. Congenital dilatation of bile ducts

- type I: dilatation of the common bile duct

- large saccular

- small localized

- diffuse fusiform

type II: diverticulum of the common bile duct or

gall-bladder

- type III: choledochocoele

- type IV: multiple intrahepatic and extrahepatic

dilatations (Caroli's disease)

- type V: fusiform intrahepatic and extrahepatic dilatations 
Although nothing is known regarding heredity, these morphological findings suggest the adult type of polycystic disease of the liver in these 3 cases; in addition, hereditary factors in the dog may differ from those in man. Descriptions in veterinary literature (Jubb and Kennedy, 1970; Jones and Hunt, 1983), although often incomplete, suggest that solitary cysts and the adult type of polycystic disease occur in the dog.

The childhood type of polycystic disease of the liver in man is inherited in an autosomal recessive manner and is characterized morphologically by polycystic kidneys type I according to Potter and Craig (1975) and cystic lesions in the liver with a varying degree of fibrosis. The liver does not appear abnormal macroscopically. Histologically, there is a striking increase in the number of biliary channels that arise in portal areas and extend irregularly and deeply into the lobules. The classification into 4 types: 1. perinatal, 2. neonatal, 3 . infantile and 4. juvenile (Blyth and Ockenden, 1971) is based on the decreased cystic involvement of the kidneys and increased hepatic fibrosis with advancing age. Congenital dilatation of the bile ducts types IV and V in man is characterized by cystic and fusiform dilatation, respectively, of the intra- and extrahepatic bile ducts; bilirubin calculi may be found in the lumen of these ducts. Usually the ducts show a varying degree of fibrosis due to a severe secondary ascending cholangitis. According to Caroli (1973) the dilatation of the common bile duct is the physiological response to primary lesions of the intrahepatic biliary tree. In congenital dilatation of the bile ducts types IV and $\mathrm{V}$, several forms of kidney disease are described, i.e. polycystic kidneys type I and medullary cystic spongiosis. Congenital hepatic fibrosis is characterized by severe portal fibrosis, with many small dilated bile ducts; it may be associated with renal medullary cystic spongiosis. However, the severe fibrosis present in the juvenile form of childhood polycystic disease of the liver and in congenital dilatation of bile ducts types IV and V, complicated by ascending cholangitis, may lead to a similar liver morphology to that in congenital hepatic fibrosis.

The liver and kidney lesions in our case 7 and in the Cairn terrier pups described by McKenna and Carpenter (1980) are basically identical. Both showed fusiform dilatation of the intrahepatic and extrahepatic bile ducts and polycystic kidneys type $\mathrm{I}$. The presence of bilirubin calculi in the dilated bile ducts, the severe dilatation of the extrahepatic bile ducts and the fibrosis in the kidneys in our dog may be associated with its long survival (14 months) compared with the Cairn terrier pups. The morphological lesions in these canine cases warrant the diagnosis "congenital dilatation of the bile ducts type V'. A marked feature is the absence of ascending cholangitis in our dog in comparison with the human situation, which reflects the resistance of the dog to ascending cholangitis and has also been reported in other forms of biliary disease in the dog.

The considerable overlap between the childhood type of polycystic disease of the liver, congenital dilatation of bile ducts types IV and V and congenital hepatic fibrosis, as mentioned in the human literature, probably results from inappropriate criteria used for their differentiation. For these diseases neither the degree of fibrosis of the liver nor the type of kidney lesions seems specific.

A better delineation of the syndromes may be the presence or absence of 
extrahepatic lesions and the nature of the cystic lesions. The childhood type of polycystic disease of the liver may thus be defined as microscopical dilatation and increase of intrahepatic bile ducts in the liver with varying degrees of fibrosis (Jørgensen, 1972) and without lesions of the extrahepatic bile ducts; ascending cholangitis is rare in these cases. In this definition, congenital hepatic fibrosis is part of the syndrome of the childhood type of polycystic disease of the liver, as already suggested by Jorgensen (1972). Congenital dilatation of the bile duct types IV and V may then be defined as multiple or fusiform dilatation of intrahepatic bile ducts associated with dilatation of the extrahepatic bile ducts, often accompanied in man by secondary ascending cholangitis.

\section{SUMMAR Y}

Seven canine cases of cystic disease of the liver are described. They included 3 cases with solitary cysts, 3 with the adult type of polycystic disease of the liver and one with congenital dilatation of the bile ducts type $V$, i.e. fusiform dilatation of the intrahepatic and extrahepatic bile ducts. These findings are discussed with respect to the morphology and classification of human cases of cystic liver diseasc.

\section{REFERENCES}

Blyth, H., and Ockenden, B. G. (1971). Polycystic disease of kidneys and liver presenting in childhood. Fournal of Medical Genetics, 8, 257-284.

Caroli, J. (1973). Diseases of the intrahepatic biliary tree. Clinics in Gastro-enterology, 2, $147-161$.

Hadad, A. R., Westbrook, K. C., Campbell, G. S., Caldwell, F. T., and Morris, W. D. (1976). Congenital dilatation of the bile ducts. The American Journal of Surgery, 132, 799-804.

Ishak, K. G., and Sharp, H. L. (1979). In Pathology of the Liver. R. N. M. McSween, P. P. Anthony, P. J. Scheuer, Eds, Churchill Livingstone, Edinburgh, pp. 75-80.

Jones, T. C., and Hunt, R. D. (1983). Veterinary Pathology, 5th Edit. Lea and Febiger, Philadelphia, p. 1428.

Jørgensen, M. (1972). Three-dimensional reconstruction of intrahepatic bile ducts in a case of polycystic disease of the liver in an infant. Acta Pathologica et Microbiologica Scandinavica, A, 80, 201-206.

Jubb, K. V. F., and Kennedy, P. C. (1970). Pathology of Domestic Animals, 2nd Edit. Academic Press, New York, p. 195.

McKenna, S. C., and J. L. Carpenter (1980). Polycystic disease of the kidney and liver in the Cairn terrier. Veterinary Pathology, 17, 436-442.

Murray-Lyon, I. M., Ockenden, B. G., and Williams, R. (1973). Congenital hepatic fibrosis - is it a single clinical entity? Gastroenterology, 64, 653-656.

Nakanuma, Y., Terada, T., Ohta, G., Kurachi, M., and Matsubara, F. (1982). Caroli's disease in congenital hepatic fibrosis and infantile polycystic fibrosis and infantile polycystic disease. Liver, 2, 346-354.

Potter, E. L., and Craig, J. M. (1975). Pathology of the Fetus and the Infant, 3rd Edit. Yearbook Medical Publishers Inc., Chicago, pp. 393-407. 